\title{
Preferential Growth of Single-Walled Carbon Nanotubes on Silica Spheres by Chemical Vapor Deposition
}

\author{
Weiwei Zhou, Yan Zhang, Xuemei Li, Shiling Yuan, Zhong Jin, Junjian Xu, \\ and Yan $\mathrm{Li}^{*}$
}

\section{Supporting materials}

\section{The influence of the flow of $\mathrm{CH}_{4}$ on the growth of SWNTs}

In our previous experiments of SWNTs synthesized on the flat silicon substrate, the flow of 500 sccm of $\mathrm{CH}_{4}$ usually was applied, whereas, seldom SWNTs were obtained on the silica surface, so we tried to reduce the flow of $\mathrm{CH}_{4}$ to $400 \mathrm{sccm}$ or 300 sccm. When the flow of 300 sccm of $\mathrm{CH}_{4}$ was used, a great number of SWNTs can be reproducibly obtained on all sized silica spheres, and the effect is more prominent for smaller-sized silica spheres. SEM images (figure S1) show the typical result of the same silica sphere samples after CVD processes with different flow of $\mathrm{CH}_{4}$. It is well known that in methane CVD process methane first absorbs on the surface of matrix and then decomposes with the assistance of catalyst. When the dispersion of silica spheres is dropped on the silicon wafer, the silica spheres prefer to pile together during the solvent evaporation, so in CVD process, adequate time is necessary for $\mathrm{CH}_{4}$ to enter the limited space between silica spheres and be absorbed on the surface of silica spheres. When we reduced the flow of $\mathrm{CH}_{4}$ furthermore, the amount of SWNTs decreased because the partial pressure of $\mathrm{CH}_{4}$ is too low to maintain the whole reaction.
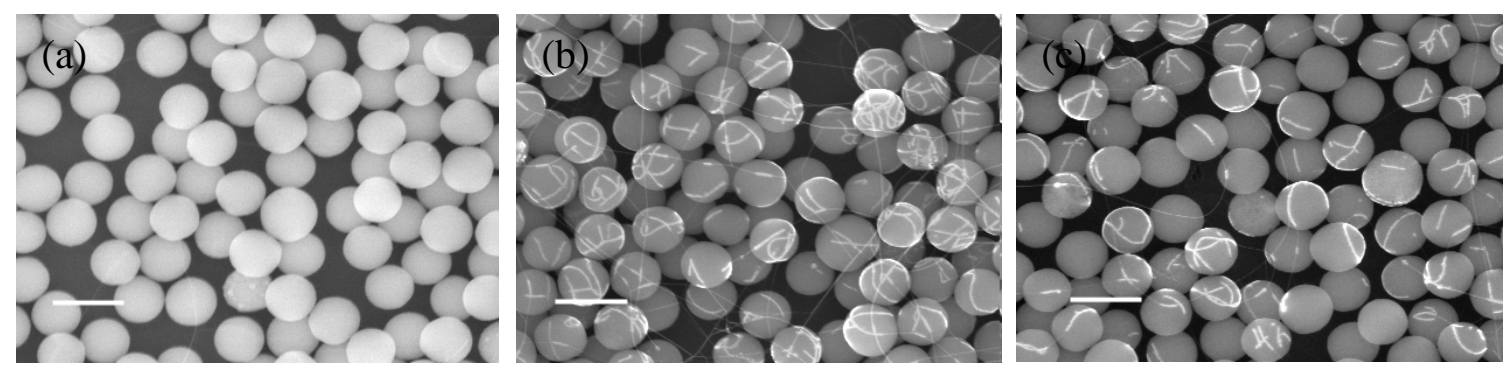

Figure S1. SEM images of SWNTs grown on silica spheres of 350nm in diameter using CH4 with the flow rate of 400sccm (a), 300sccm (b), and 200sccm, respectively, during CVD growth. 


\section{Raman spectra of SWNTs on silica spheres of different sizes.}

The RMB for SWNTs can be found in the Raman spectra (figure S2) of the six samples with different size. It is noticeable that the intensity ratios of $G$ band to $D$ band are all very high in all these samples, which indicates that the SWNTs we got are of high purity and little defects formed in the tube walls. We noticed that there are high backgrounds in figure S2d and S2e. We speculate that there is much scatter for incident laser because the diameters of the two kinds of silica spheres are close to the excitation wavelength of the Micro-Raman.
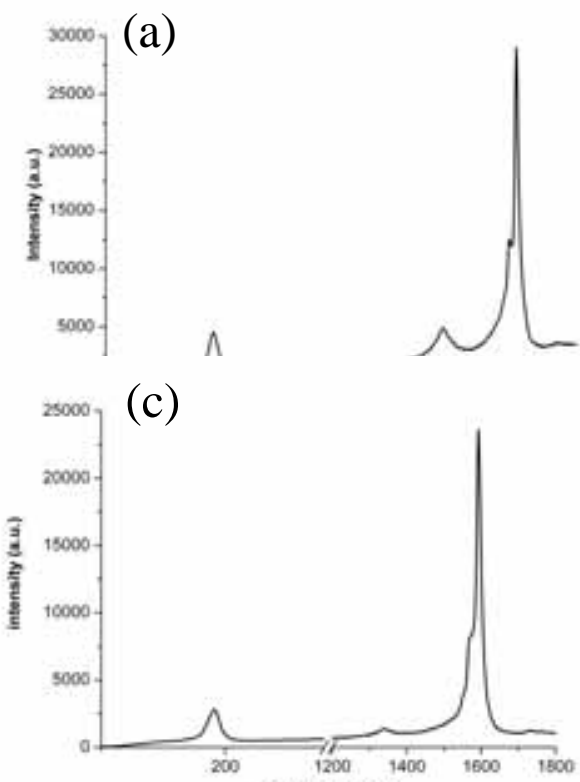

(e)

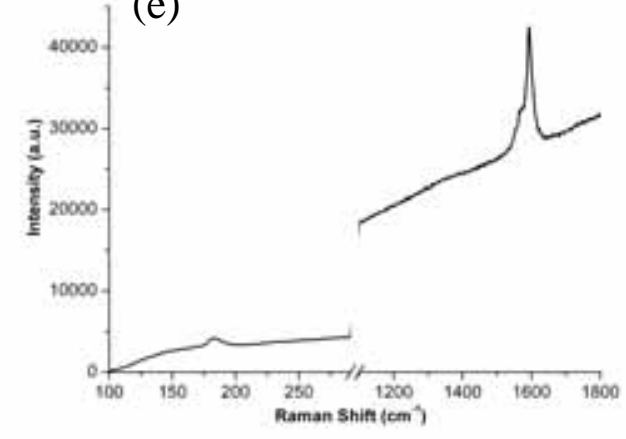

(b)

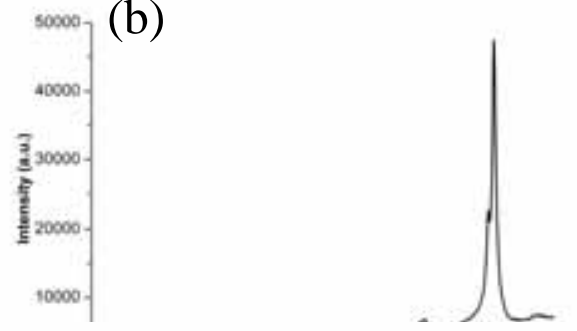

(d)

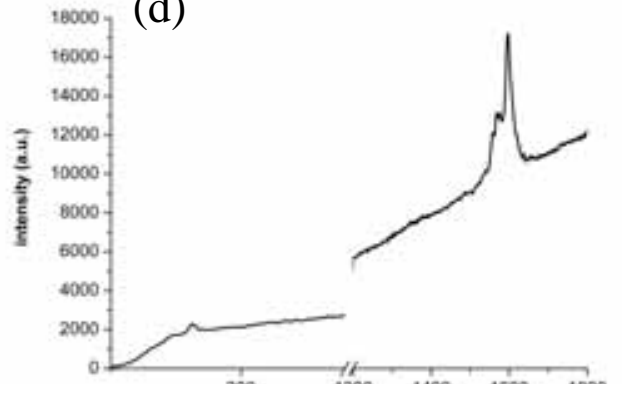

(f)

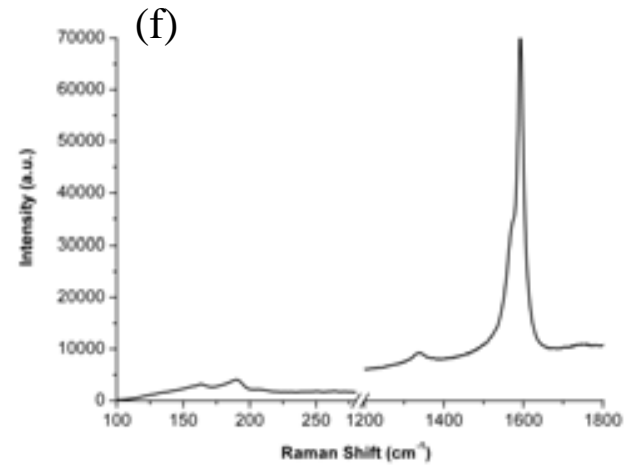

Figure S2. Raman spectra of SWNTs grown on silica spheres of $300 \mathrm{~nm}, 350 \mathrm{~nm}$, 400nm, 500nm, 580nm, and 680nm in diameter (a to f), respectively. 